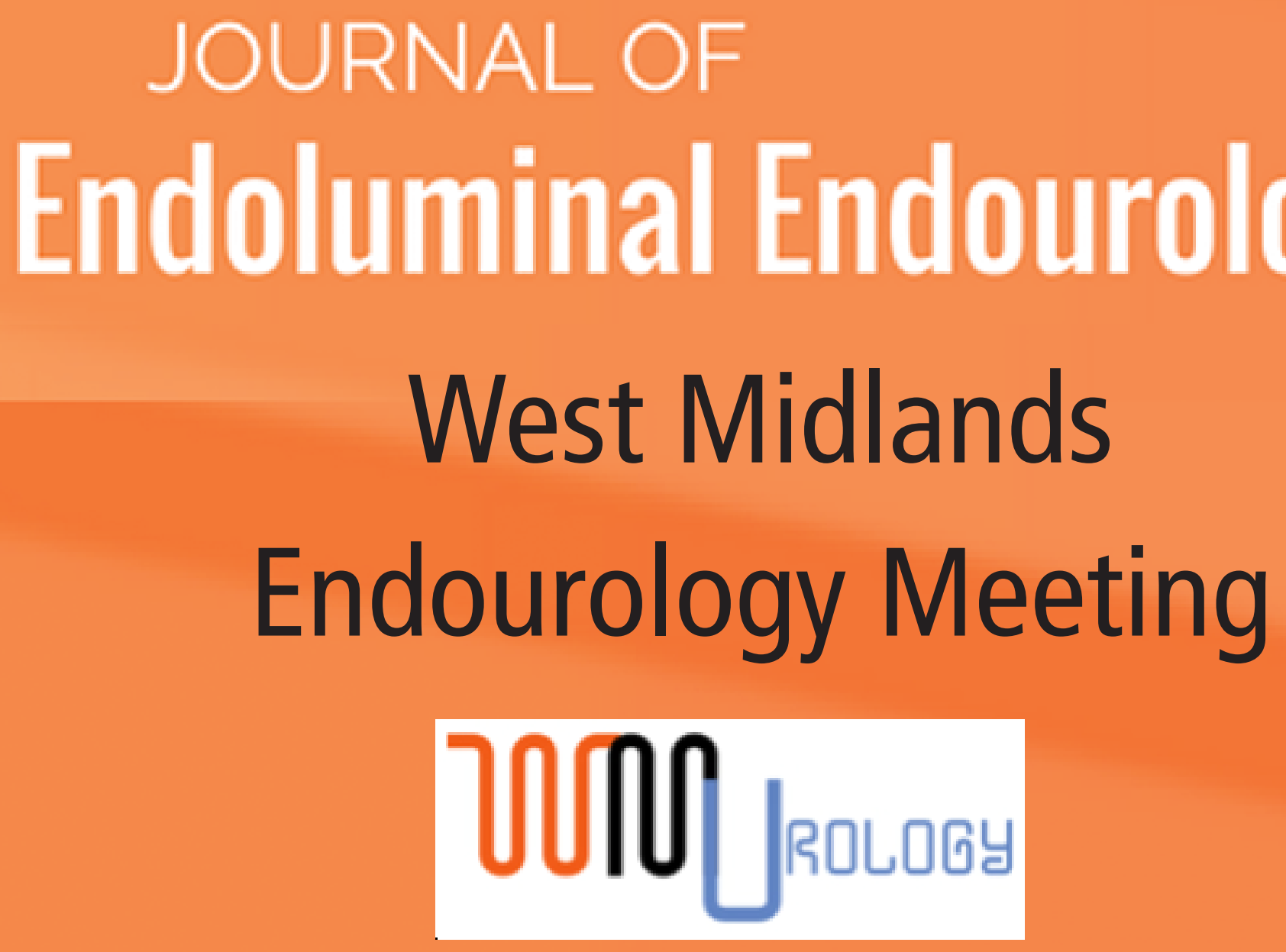




\section{The Impact of COVID-19 on Training and Delivery of Urology Services in the West Midlands}

N Eli, L Siriwardena, M Moore, D Mak and T King on behalf of the West Midlands Research Collaborative Group

\section{Introduction}

The SARS-CoV-2 pandemic has affected 246 million people since it was first reported in December 2019. It has profoundly affected the way we deliver health care and consequently, training opportunities across all surgical specialities have been severely impacted.

\section{Methods}

A cross sectional multicentre study across the West Midlands assessed the impact of COVID-19 on urology training and service delivery, by comparing April to September 2019 pre-COVID-19 (PC) and April to September 2020, COVID-19 (C). 16 trainees' surgical logbooks in the region were compared during the $\mathrm{PC}$ and $\mathrm{C}$ periods. To assess service delivery during the first wave, questionnaires were prospectively collected from the 12 training units in the West Midlands.

\section{Results}

Trainee indicative operations were analysed using the Pair T-Test (table1). Statistically significant results for; LUTS surgery $(\mathrm{p}=0.0011)$, TRUS biopsy $(\mathrm{p}=0.0102)$, urodynamics $(\mathrm{p}=0.0173)$, and adult circumcision $(p=0.0139)$. Scrotal procedures were insignificantly different in keeping with emergency surgery. Although statistically insignificant, some oncology procedures did have a perceptible change; TURBT and nephrectomy. Andrology, female, and paediatric surgery were all in low numbers.

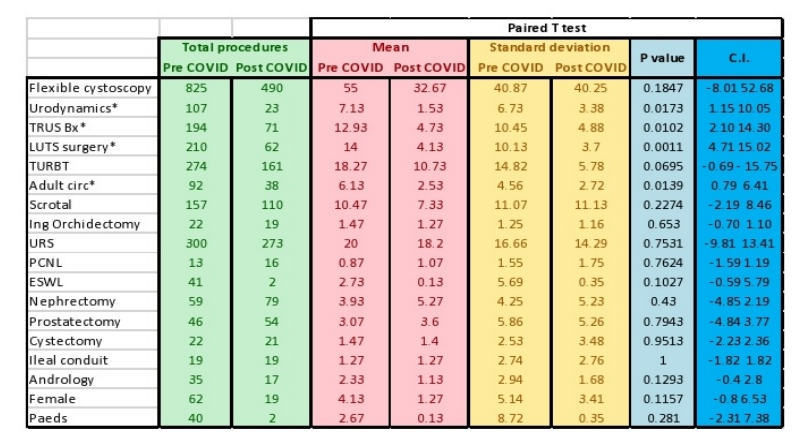

Analysis of seven training units showed significant $(\mathrm{p}<0.05)$ reduction in indicative operations from 4 units during the COVID study period.

Questionnaire responses revealed one unit stopped all elective activity whilst another continued to perform all oncological operation during April 2020. By September 2020 all oncological operations, bar one unit, had recommenced. Benign operations were curtailed in all units during the pandemic.

\section{Conclusion}

This study demonstrates that COVID-19 has significantly impacted urology training and service delivery across the region. 


\title{
Robot-Assisted Radical Cystectomy: A Complete Audit Cycle of Our First 120 Cases
}

\author{
N Eli, M Dowdeswell, W Elsayed, D Mak, P Cooke \\ Royal Wolverhampton NHS Trust
}

\section{Introduction}

There are 10,200 new cases of bladder cancer diagnosed yearly in the UK accounting for 3\% of all new cancer diagnoses. Robot-assisted radical cystectomy (RARC) is a minimal invasive treatment option for muscle invasive and high risk bladder cancer with a rising uptake. Between 2017 and 2019, RARC accounted for $40.6 \%$ of all cystectomies performed in the UK.

We compared our outcomes with the best practice of RARC set by the Pasadena consensus panel in 2015.

\section{Methods}

A review of the prospective database of all RARC performed by two surgeons at Royal Wolverhampton NHS Trust between May 2013 and October 2020 was conducted. Operative and oncological outcome data were collected from Clinical Web Portal and Somerset cancer Register and analysed with Microsoft Excel.

\section{Results}

A total of 120 RARC were performed in the study period, 50 cases were analysed in the first audit cycle and 70 cases in the second loop. The mean age was 70 years, $84 \%$ were men and $70 \%$ were ASA II or less. The median operating time was 372 minutes and the median blood loss was $240 \mathrm{mls} .83 \%$ of the urinary diversion was intra-corporeal and $89 \%$ was Ileal conduit diversion. The median length of stay was 8 days; Clavien-Dindo complication grade III or more was $15 \% .87 \%$ of our patients are still alive.

\section{Conclusions}

RARC is a safe option for the radical management of bladder cancer. Our results are consistent with the Pasadena consensus recommendations and demonstrate improvement in surgical outcomes between the audit cohorts.

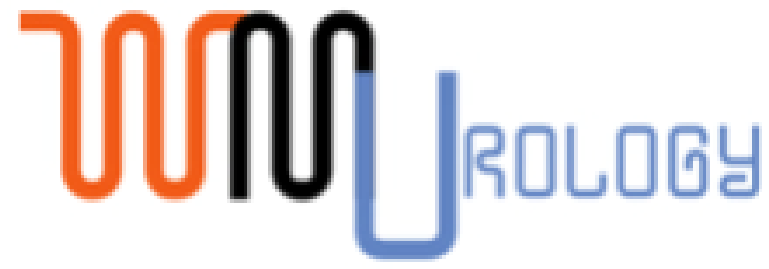

JELEU Vol 4(4):e1-e11; December 20, 2021.

This article is distributed under the terms of the Creative Commons Attribution-

Non Commercial 4.0 International License 


\title{
A 10-year Review of Orchidectomies at George Elliot Hospital: Our Changing Practice
}

\author{
Z Kasmani, A Damola, B Ogar, I Apakama
}

\section{Introduction}

Orchidectomies are performed for a variety of reasons including the management of testicular cancer. Suspected testicular cancer should be imaged with USS before surgery to confirm diagnosis. We aimed to review all the orchidectomies done at our trust over a 10-year period to assess our hospital's practice in line with NICE guidance on the diagnosis and management of testicular cancer and also the guidelines of the west midland expert advisory group for urological cancer.

\section{Methods}

We reviewed all patients that had orchidectomies between 2009 and 2019. We reviewed the USS reports, blood tumour markers, demographic such as race, age and smoking status of patients and the outcomes of the histology report.

\section{Results}

Overall our USS had a sensitivity of $97.7 \%$ but a specificity of only $69.2 \%$, with 12 orchidectomies were benign. 38\% (57/150) of orchidectomies were done for surgical castration of prostate cancer between 2009 and 2019. 78.6\% of these were done between 2009 and 2014, with $21.4 \%$ being done between 2014 and 2017, and with no surgical castration done after 2017. 38/43 patients with testicular cancer (65\%) had normal tumour markers.

\section{Results}

In our practice, the sensitivity of USS was $97 \%$ compared to the standard of $90 \%$. However, we had a lower specificity which resulted in 12 benign orchidectomies. We shall endeavour to improve the specificity of our USS. We also highlight the impact hormone modulating drugs have had in reducing the need for surgical castration, now almost confined to history at our hospital.

\begin{tabular}{|c|c|c|c|c|c|c|}
\hline \multirow[b]{2}{*}{ Pathology } & \multicolumn{3}{|l|}{ Germ cell } & \multicolumn{2}{|l|}{ Other } & \multirow[b]{2}{*}{ Castration } \\
\hline & Seminoma & $\begin{array}{l}\text { Non- } \\
\text { seminoma }\end{array}$ & $\begin{array}{l}\text { Mixed } \\
\text { germ } \\
\text { cell }\end{array}$ & $\begin{array}{l}\text { Sex-cord } \\
\text { (stromal) }\end{array}$ & Lymphoma & \\
\hline $\begin{array}{l}\text { Number of } \\
\text { patients }\end{array}$ & 22 & 8 & 8 & 3 & 2 & 57 \\
\hline $\begin{array}{l}\% \\
\text { detected } \\
\text { on USS }\end{array}$ & $95.5 \%$ & $100 \%$ & $100 \%$ & $100 \%$ & $100 \%$ & NA \\
\hline $\begin{array}{l}\text { Average } \\
\text { age }\end{array}$ & 41.3 & 42.5 & 38.2 & 41.7 & 68 & 77.7 \\
\hline
\end{tabular}




\title{
A Prospective Multi-institutional Study Using the Transurethral Catheterisation Safety Valve (TUCSV $\odot$ ) for the Prevention of Catheter Balloon Inflation Injury of the Urethra
}

\author{
A. Matkowski \\ Hereford and Irish Hospital Groups
}

\section{Introduction}

Catheter balloon injuries (CBI) of the urethra are avoidable iatrogenic injuries which occur in just over $1 \%$ of hospitalised male inpatients. The transurethral catherization safety valve (TUCSVC) is a novel safety device designed to prevent CBIs. The safety device allows fluid in the catheter system to vent through a pressure relief valve if the catheter's anchoring balloon is inflated in the urethra. The aim of this multi-institutional clinical study was to prospectively assess the TUCSV's ability to prevent urethral CBI over a 3-month study period.

\section{Methods}

The incidence of CBI was recorded in 2 Irish hospital groups and UK based hospital over a 3-month period. Following this the TUCSV was introduced for urinary catheterisation in a variety of hospital settings in the primary institution of the hospital groups over a 3-month study period in 2021. The safety valve is compatible with all commercially available catheters. Data were recorded using a 7-item data sticker with a scannable QR code. 'Venting' through the safety valve during catheterisation was indicative of a urethral injury prevented. Any CBIs referred to the Urology team on call were recorded.

\section{Results}

In total, 729 urethral catheterisations were carried out using the TUCSV, with 16 (2.2\%) episodes of TUCSV venting recorded. There were no urethral injuries in these patients. In the same period, 14 urethral CBIs were recorded where the TUCSV was not utilised. This suggests that the true incidence of CBI is substantially greater than that based on cases referred to Urology only. The injury rate was 25/ 4248 or $5.9 / 1000$ catheterisations or $10.4 / 1000$ male catheterisations.

\section{Conclusions}

Iatrogenic CBIs represent a significant cause of patient morbidity. The TUCSV has the potential to eliminate CBI of the urethra if widely adopted. It represents a simple, effective and innovative solution to this recurring problem applicable to all patient cohorts in all catheterisation settings.

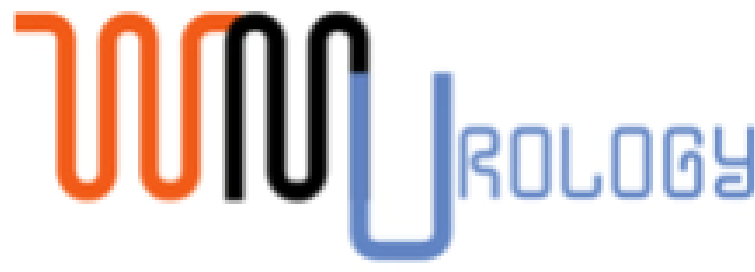

JELEU Vol 4(4):e1-e11; December 20, 2021.

This article is distributed under the terms of the Creative Commons Attribution-

Non Commercial 4.0 International License 


\title{
Do Renal Mass Biopsies Influence Patient Management? A Case Series Study in a Tertiary Hospital
}

L Kretzmer

University Hospital Birmingham

\begin{abstract}
In recent years renal mass biopsy (RMB) has been increasingly adopted to guide management in patients with renal lesions. Indications include the evaluation of renal masses or cysts, and diagnosis prior to initiating targeted chemotherapy or ablation. A case series of 232 RMBs was collected from 2009 -2020. Inclusion criteria were RMBs taken from native kidneys.

Participants' mean age was 64.7 years ( $\mathrm{SD}=13.57$ years), with $67 \%$ being male. The diagnostic rate of RMB was $78.45 \%(n=182 / 232)$. Whether masses were solid or cystic did not influence diagnostic rate $(\mathrm{p}=.94143)$. Positive predictive value (PPV) of RMB compared with surgical histology was .8775 . Only $1.3 \%(n=3 / 232)$ of cases had significant complications.

Patients with non-diagnostic biopsies were more likely to be managed with surveillance alone (56.0\% vs $32.4 \% ; \mathrm{p}<.05)$. Diagnostic RMBs did not statistically influence whether patients had surgical management $(p=.188914)$. Of non-diagnostic RMBs, $100 \%(n=7 / 7)$ of patients with surgical histology had renal cell carcinoma (RCC).

RMB has specific implications in two special populations: In small renal masses, PPV of RMB increased to $1.0 .16 .7 \%(n=3 / 18)$ of patients diagnosed with oncocytoma at RMB had a diagnosis of RCC. RMBs are safe with a high diagnostic rate. However it seems to not influence definitive patient management. Clinicians should be wary when dealing with special populations. Results must be viewed in the context of the patient's history and imaging, and management should be determined using a multidisciplinary approach.
\end{abstract}

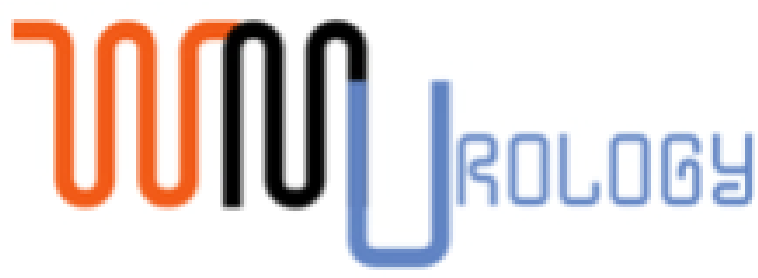

JELEU Vol 4(4):e1-e11; December 20, 2021.

This article is distributed under the terms of the Creative Commons Attribution-

Non Commercial 4.0 International License 


\title{
The Role of Prostate-Specific Antigen Density (PSAD) in the Prediction of High- Grade Disease and Extracapsular Extension in Prostate Cancer
}

\author{
D Saleh ${ }^{1}$, A Afzal $^{1}$, U Nawaz ${ }^{1}$, MO Kitchen ${ }^{1,2}$, AM Abd El-Baky ${ }^{1}$ \\ ${ }^{1}$ Urology Department, University Hospitals of Birmingham NHS Trust, UK. \\ ${ }^{2}$ School of Medicine, Keele University, UK.
}

\section{Introduction}

Multiple factors help risk-stratify patients and guide management in prostate cancer, including prostatespecific antigen density (PSAD). PSAD forms part of Uro-radiologist assessment for Likert scoring of mpMRI scans; when raised $(>0.15)$, is a strong predictor of prostate cancer diagnosis. However, few studies describe a prognostic role of PSAD. We assessed correlations between PSAD and prostate cancer stage/grade, that might improve patient management even before prostate biopsy.

\section{Patients and Methods}

We retrospectively identified men undergoing radical prostatectomy at our University Teaching hospital between 2016 and 2019. Clinical and demographic data were collected, including PSA, mpMRI findings, prostate biopsy histology, and prostatectomy histology. Receiver-operated characteristic (ROC) analysis determined optimal PSAD value that predicted Gleason score eight or above, and T3 tumours.

\section{Results}

335 patients were included; median age was 65.8 years (range $44-79$ ). Median iPSA was 7.35 (range 0.96 - 53.00). Median PSAD was 0.18 (range 0.01 - 1.80). mpMRI estimated prostate volume median was $40.0 \mathrm{~cm} 3$ (range $9.1-212.0$ ).

ROC analyses identified PSAD $\geq 0.18$ as the best cut-off for Gleason $\geq 8$ (AUC 0.5523) and T3 staging (AUC 0.6410). Furthermore, 14/17 patients upgraded from Gleason 7 to $\geq 8$ had PSAD $\geq 0.18$, and 59/97 patients upstaged from $\mathrm{T} 1$ or $\mathrm{T} 2$ to $\mathrm{T} 3 \mathrm{had} \mathrm{PSAD} \geq 0.18$.

\section{Conclusions}

PSAD merits further investigation as a prognostic tool in prostate cancer. It is possible that PSAD could be used as an adjunct to patient and tumour factors to inform patients of their risks of aggressive disease and inform treatment decisions.

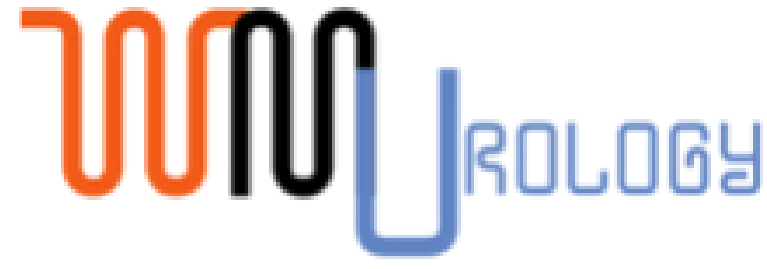




\title{
Sperm Banking Prior to Radical Orchidectomy: A Retrospective Consecutive Case Series Audit
}

\author{
A Sultana ${ }^{1}$, C Desai ${ }^{1}, \mathrm{~N} \mathrm{Ibrahim}^{1}$, A Ehsanullah ${ }^{1}$, S Dunk $^{1}$, M Kitchen $^{1,2}, \mathrm{~K} \mathrm{Ho}^{1}$ \\ ${ }^{1}$ Heart of England Foundation NHS Trust, Birmingham, UK \\ ${ }^{2}$ School of Medicine, Keele University, Staffordshire, UK
}

\section{Introduction}

Testicular cancers can have high metastatic potential and rapid tumour doubling time. Management is therefore expedient staging, serum tumour marker evaluation, and radical orchidectomy. To best preserve fertility/paternity rates, sperm-banking should be performed prior to orchidectomy (EAU guidelines recommendation), but anecdotally, many centres and Urologista favour expediency over holistic care (sperm banking). We assessed our practice of pre-operative counselling.

\section{Patients and Methods}

95 patients underwent radical orchidectomy for suspected testicular cancer between January 2015 and January 2020 at our University Teaching Hospital. Peri-operative clinical and demographic data were collected, including discussion of sperm-banking.

\section{Results}

39/95 patients were offered sperm-banking pre-orchidectomy (20/39 completing the process). Reasons for patient declining sperm-banking where offered included patient age (one case) and already having completed a family (two cases). 4/95 patients were offered sperm-banking post-operatively. Spermbanking was therefore not offered (or offered but not documented) in 56/95 cases.

Importantly, the average time from referral to orchidectomy in patients undergoing sperm-banking was similar (21.1 days), to those not undergoing sperm-banking (average 21.2).

\section{Conclusion}

There is variability in counselling prior to radical orchidectomy. It is evident some clinicians do not offer sperm-banking, most likely due to fear of delays to orchidectomy, however, sperm-banking does not delay surgery and therefore should be discussed with all men undergoing radical orchidectomy. Overall however, 21 days from referral to orchidectomy was considered too long, and led a streamlined pathway of review in Andrology clinic for sperm-banking counselling and urgent orchidectomy, rather than historic practice of US scan discussion at MDT prior to clinical examination.

\section{ปก) |robagy}

JELEU Vol 4(4):e1-e11; December 20, 2021.

This article is distributed under the terms of the Creative Commons Attribution-

Non Commercial 4.0 International License 


\section{Outcome Audit of Ureteroscopic Stone Surgery: Does Longer Operative Time $>90$ mins Result in Higher Risk of Sepsis?}

T Burnhope, S Yallappa, M El-Hadi, D Nyanhongo, V Koo

Worcestershire Hospitals

\section{Introduction}

As ureteroscopic technology and techniques continue to improve, larger and more complex stones are being treated endoscopically by the endourologist. As such, a longer operative time could potentially result in increased risk of post-operative sepsis.

\section{Methods}

All ureteroscopies performed for stone management by a single surgeon were included and follow-up procedures were excluded. Retrospective analysis of the patient, stone, treatment and stent data were obtained. The cases were sub-grouped according to Short $(<90 \mathrm{mins})$ and Long ( $\geqslant 90$ mins) of operative time.

\section{Results}

Out of 272 cases, the Short( $\mathrm{n}=182)$ vs Long(n=90) groups were comparable in terms of mean age, BMI and ASA scores. Target stone site distribution in Short vs Long groups was Renal (35.8\% vs 56.7\%), staghorn $(0.5 \%$ vs $20 \%)$, calyceal diverticulum $(3.3 \%$ vs $4.4 \%)$ and ureteric $(60.4 \%$ vs $18.8 \%)$ respectively. The Short group had lower positive pre-operative MSU rates (7.6\% vs $21.1 \%$ ), higher preoperative stent rates $(36.2 \%$ vs $43.3 \%)$ and smaller mean stone size $(8.4 \mathrm{~mm}$ vs $18.1 \mathrm{~mm})$.

The Short group had a mean operative time of 46mins vs $117 \mathrm{mins}$, used less flexible ureteroscopy as primary instrument (52.7\% vs $91.1 \%)$ and lower post-operative stent rates $(20.3 \%$ vs $98.8 \%)$. The mean length of hospital stay were similar 1.2 days vs 1.5 days. The Short group had 4 patients vs 3 patients who developed sepsis post-operatively, and this was not found to be statistically significant.

\section{Conclusion}

Although the Long group had expectedly larger and more complex stones with higher pre-operative UTI, the post-operative sepsis risk was not increased despite longer operative time.

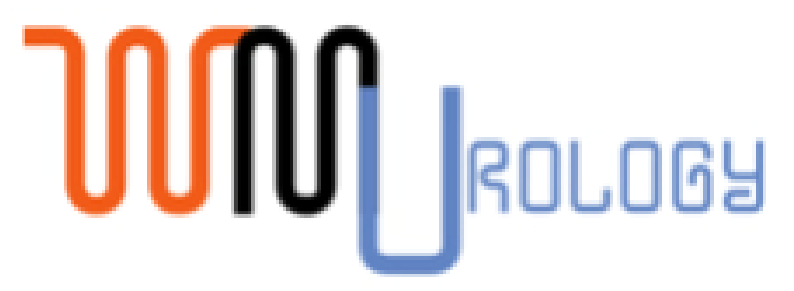

JJELEU Vol 4(4):e1-e11; December 20, 2021.

This article is distributed under the terms of the Creative Commons Attribution-

Non Commercial 4.0 International License 
2021 West Midlands Endourology Meeting

\title{
Audit of Testicular Cancer Management in the West Midlands Deanery
}

\author{
A Kanthabalan ${ }^{1}$ West Midlands Research Collaborative ${ }^{2}$ I Wharton ${ }^{3}$ \\ ${ }^{1}$ Queen Elizabeth Hospital, Mindelsohn Way, Birmingham B15 2TH \\ ${ }^{2}$ West Midlands Region \\ ${ }^{3}$ University Hospital Coventry \& Warwickshire
}

Keywords: Testicular Cancer, Radical inguinal Orchidectomy, Sperm Banking, Tumour markers

\section{Background}

Testicular cancer represents 5\% of urological tumours ${ }^{\mathrm{i}}$. Current NICE Guidelines ${ }^{\mathrm{ii}}$ state that patients with suspected testicular cancer should be referred on the 2 week wait pathway, with urgent ultrasound imaging serum tumour markers and be offered prosthesis and sperm banking prior to surgery. Our aim was to audit clinical practice and histopathological outcome within the West Midlands Deanery.

\section{Methods}

All Urology departments in the West Midlands Deanery were requested to complete data covering a retrospective five year period (2015-2020) on patients aged 16 years and over referred for suspected testicular cancer. Parameters assessed included risk factors, ultrasound findings, tumour markers, offering of prosthesis insertion and sperm banking, histology and staging.

\section{Results}

10 Urology departments participated and 824 patients were included. Of those aged between 16-50 24\% $(133 / 561)$ were offered sperm banking. 7\% (30/428) were reported to have completed child bearing age and therefore not offered sperm banking. However, for those not offered, there was poor documentation. Of all patients offered sperm banking $47.7 \%(82 / 172)$ took up the offer.

$41.9 \%(345 / 824)$ of patients were offered prosthesis, of which $41.5 \%(143 / 345)$ took up the offer. Overall embryonal cancers were the most common (50.2\%), 14.3\% were mixed germ cell cancers. 59.2\% were Stage I tumours.

\section{Conclusion}

This audit reveals that the majority of patients within the West Midlands there is significantly poor documentation regarding offering sperm banking and prosthesis at time of consent for radical orchidectomy. Histopathological data outcomes reveal early stage of testicular cancer.

ihttps://uroweb.org/wp-content/uploads/EAU-Guidelines-on-Testicular-Cancer-2019-1.pdf

iihttps://www.england.nhs.uk/mids-east/wp-content/uploads/sites/7/2018/05/guidelines-for-the-management-of-testicular-cancer.pdf

JELEU Vol 4(4):e1-e11; December 20, 2021.

This article is distributed under the terms of the Creative Commons Attribution-

Non Commercial 4.0 International License 


\section{Putting Diversity on Paper - An Audit of Ethnicity and Diversity Information on Acute Urology Admission}

Michael Keunhwi Ahn, Sheriff Akande, Oluwatobiloba Odeyemi, Ayo Kalejaiye

Department of Urology, Birmingham Heartlands Hospital, University Hospitals of

Birmingham NHS Foundation Trust

\section{Background}

In the United Kingdom, we are living in a multicultural and multi-ethnic society. Birmingham cherishes a diverse community, where nearly half of the population are declared as Black, Asian, and minority ethnic (BAME) groups. ${ }^{1}$ The most recent NICE guideline highlights the importance of BAME patient representation and care. ${ }^{2}$ Good record-keeping is paramount to achieve this and to provide ongoing support.

\section{Results}

This audit is in the process of being registered with the local office. We are hoping to expand this audit in the future to include the documentation of the patient's spoken language and offer of translation services to improve patient experience.

\section{Conclusion}

With the increasing awareness of promoting cultural diversity amongst BAME groups, efforts must be made to keep good record and provide help and support to patients with language barriers by providing necessary support services. This audit is the first step forward in our trust to promote this and advocate for our diverse patient population.

1. https://www.nomisweb.co.uk/census/2011/QS201EW/view/1946157186?cols=measures

2. https://www.nice.org.uk/guidance/qs167/documents/draft-quality-standard

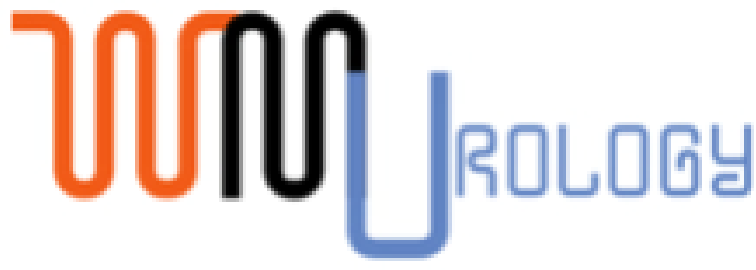

JELEU Vol 4(4):e1-e11; December 20, 2021.

This article is distributed under the terms of the Creative Commons Attribution-

Non Commercial 4.0 International License 